\title{
Entretien : pour une intégration des datadéontologues dans les équipes communication
}

\author{
Assaël Adary, Président / ceo, \\ Occurence \\ assael.adary@occurence.fr \\ Université Bourgogne Franche-Comté \\ jean-claude.domenget@univ-fcomte.fr
}

Jean-Claude Domenget, Maître de conférences HDR, 
1. Bonjour Assaël Adary, pouvez-vous vous présentez en quelques mots et préciser votre parcours professionnel et notamment votre expérience dans le domaine de la communication?

J'ai suivi une formation en philosophie, puis en communication au CELSA, à la Sorbonne. En 1995, j'ai créé ma structure, qui s'appelle Occurrence, un cabinet d'études et de conseil en communication. Aujourd'hui Occurrence, c'est 25 collaborateurs. Notre métier n'est pas celui d'une agence mais celui d'évaluateur. Il s'agit de diagnostiquer la performance des actions de communication. Nous avons donc un regard qui porte tout de suite sur le sujet de l'éthique : le fait de n'être pas juge et parties, c'est-à-dire que lorsqu'on est diagnostiqueur, on ne peut pas le matin diagnostiquer et l'après-midi vendre nos services au même client. Ce que je décris là ne va pas de soi car aujourd'hui la plupart des grands instituts d'études appartiennent à des grands groupes. L'institut CSA appartient à Bolloré donc à Havas, TNS-Sofres Kantar à WPP, etc. Il y a des vrais sujets d'éthique dans le métier du diagnostic.

Afin de préciser vos propos, en quoi consiste un diagnostic de mesure de performance?

Nous ne mesurons pas des outils mais leurs effets. Cela sous-entend de définir en amont les objectifs poursuivis. Cela peut paraître très basique. C'est ce qu'apprennent les étudiants de communication, en L3, définir les objectifs et les publics mais dans les faits, par l'intermédiaire de l'évaluation, notre travail revient à requestionner les fondamentaux. Cela commence souvent par une interrogation du type : que vouliezvous faire? Quels effets souhaitiez-vous produire avec votre opération digitale, vos événementiels, etc. ? Ensuite, on définit les indicateurs, on collecte les données, via des études quali/quanti, les data issus du digital, puis il y a le traitement, l'analyse, les recommandations. Côté recommandations, on va très loin, on se permet une liberté de ton. On ne privilégie aucune technique, comme le digital par exemple. Notre travail, c'est vraiment de renforcer cette boucle vertueuse : planification des actions, conduite des actions, mesure et ajustement du plan de communication de l'année d'après.

2. Vous l'évoquiez, l'éthique semble être centrale dans votre approche de la communication, en quoi consiste pour vous l'éthique en communication? Comment l'intégrez-vous dans votre pratique professionnelle au quotidien?

Pour moi, il y a deux niveaux. Un premier niveau la déontologie professionnelle qui renvoie une démarche sectorielle d'auto-régulation : le respect de chartes, des codes, etc. On oublie un peu trop souvent que nous disposons d'un certain nombre de référentiels auxquels on peut se référer ou de règlements comme le RGPD (Règlement 
154 Professionnalisation de la communication : approches sectorielles

Général sur la Protection des Données personnelles). Justement, ces normes ne sont pas assez apprises dans les écoles et universités, probablement du fait de leur aspect rébarbatif. Occurrence, par exemple, est certifié ISO 9001 et s'engage dans le respect de la norme ISO 26000 (RSE). Il est pour moi déjà très important de tenir compte de ces référentiels métiers.

Un second niveau est de l'ordre de l'éthique qui renvoie à l'individu. Il s'agit de la manière d'appréhender presque chaque geste professionnel que l'on effectue. Cela devient une routine, une «hygiène professionnelle », quelque chose auquel on ne pense plus à partir d'un certain moment. Mais néanmoins, avant que cela atteigne ce stade, il y a besoin de s'arrêter assez fréquemment sur ce qu'on fait et de se poser de vraies questions qui sont de l'ordre de l'intime. Prenons un exemple, les Réseaux Sociaux qui génèrent de nombreuses questions éthiques. Derrière de jolis mots, comme celui d'activation digitale ou «engagement des communautés », il y a du fake. Derrière l'activation digitale, il peut y avoir des choses très propres ou très sales, c'està-dire qu'on peut, sans même s'en rendre compte, engraisser une mafia ou faire travailler des enfants au Bangladesh. En plus, le digital a apporté la rapidité, l'immédiateté, ce qui court-circuite parfois le temps de l'éthique. En 10 secondes, je peux acheter 1000 faux followers sur Twitter, je peux me réfugier derrière le fait que c'est tellement rapide, tellement facile, tellement peu cher, que cela peut anesthésier mon esprit critique.

3. Justement, pourquoi ce questionnement éthique est-il si souvent évoqué aujourd'hui? Quelles évolutions du domaine de la communication peuvent expliquer ce questionnement éthique?

Le digital a conduit à rendre les choses très immatérielles. Avant, avec la publicité, il pouvait y avoir des questions sur le circuit financier, etc. La première loi Sapin, en 1993, légiférait contre les ententes entre les agences conseillères d'achat d'espaces et les médias. Il y avait une opacité, une non-transparence des marchés. Nous attendons maintenant les effets de Sapin 2 qui traite du digital. Aujourd'hui, cette préoccupation revient d'autant plus qu'il y a beaucoup d'immatérialité, plus d'instantanéité. Il y a beaucoup plus d'opacité, de boîtes noires dans notre système. La publicité programmatique par exemple, c'est une énorme machine à fabriquer de l'opacité. Plus il y a d'opacité, plus il y a de risques éthiques.

Quand un marché démontre qu'il n'arrive pas à s'auto-réguler, la loi intervient mais je pense que la loi est moins bonne que la prise de conscience individuelle. La loi, il y a toujours des plus malins pour la contourner. Alors que sa propre conscience, c'est plus difficile d'y échapper.

Existe-t-il également un niveau intermédiaire entre la loi et l'individu? 
Tout à fait, exactement. Je parlais tout à l'heure d'auto-régulation et de régulation. Pour moi, il y a trois niveaux, la régulation absolue, comme la loi Sapin, le RGPD, etc. Il y a l'auto-régulation avec des structures comme l'ARPP (Autorité de Régulation Professionnelle de la Publicité) qui est l'ancien BVP (Bureau de Vérification de la Publicité). Enfin il y aussi tout le travail qui est fait pas-à-pas par les associations, les syndicats professionnels. Je m'engage beaucoup dans l'une d'elles qui s'appelle COMENT (Communication \& Entreprise). Par exemple, nous nous sommes emparés du sujet de l'égalité femme-homme qu'on a appréhendé sous l'angle de la communication non-sexiste, en disant que la communication publicitaire est un des leviers d'apprentissage de la vie et influence la manière dont nous représentons les relations entre les femmes et les hommes. Aujourd'hui COM-ENT est reconnue par la Secrétaire d'État chargée de l'Égalité entre les femmes et les hommes Marlène Schiappa, avant elle par la Ministre Laurence Rossignol. On a sorti par exemple une check-list et un kit de la communication non-sexiste qui permettent aux annonceurs et aux agences, avant de lancer une campagne, d'évaluer de manière autonome la conformité avec les principes de communication non-sexiste. Là, on est en plein dans l'auto-régulation.

Dans le domaine éthique, COM-ENT avait conduit des réflexions sur la communication responsable...

Tout à fait, chez COM-ENT, on a engagé cette réflexion sur la communication responsable et nous avons produit un guide mais ce dernier n'est pas assez utilisé. Je le regrette car c'est un outil très utile et rare. Quand je parlais de la démarche ISO 26000... Quand elle a été créée, tout de suite nous nous sommes dit, c'est très global, il faudrait que chaque métier s'en empare pour créer son ISO 26000 « métier ». Le premier métier qui s'en est emparé, c'est la communication! Aujourd'hui, il y a donc un référentiel sur l'ISO 26000 dédié à la communication. C'était un très gros travail. On a pris tous les métiers de la communication, on a trouvé des référentiels.

Aujourd'hui, un communicant qui se pose la question de son éthique professionnelle a sous la main une somme colossale de référentiels, de règles qui peuvent l'aider ou simplement lui permettre de se poser les bonnes questions au bon moment et sur tous les plans. Comme on a pris la base de l'ISO 26000, ce n'est pas que l'écologie, la réduction du $\mathrm{CO} 2$ qui est un peu la facilité. Par exemple, un communicant décide d'imprimer en PEFC, de recycler, de faire son stand en carton, etc. et va oublier que dans nos métiers, il y a des indépendants et des free lance qui sont dans la précarité la plus totale et qu'il va payer à 120 jours fin de mois. Ce qui n'est pas responsable. Or l'avantage de l'ISO 26000, c'est qu'il y a une prise en compte de l'ensemble des aspects de la responsabilité qui permet a priori de ne pas passer à côté d'une des composantes. Et en effet, la manière de traiter le bas de la chaîne de la valeur, c'est un sujet de responsabilité et c'est un sujet profond d'éthique. 
156 Professionnalisation de la communication : approches sectorielles

Pour finir sur ce point, vous indiquiez que c'est un peu passé sous silence...

Oui, après un travail colossal, il y avait beaucoup d'associations impliquées, COMENT, l'AACC, l'UDA, etc. Ce document n'a pas eu la visibilité et l'usage souhaités. Par exemple, je ne vois aucun acheteur des grandes entreprises et des grands donneurs d'ordre valoriser dans un appel d'offres, réellement cette démarche. A la fin, je reviens toujours à l'individu. On ne le fait pas pour gagner des marchés, on le fait pour quelque chose qui est de l'ordre de l'intime, de sa conscience professionnelle. Aujourd'hui, je pense que la fonction communication dispose de l'ensemble des référentiels métiers pour travailler dans une éthique stricte mais non liberticide.

Tous ces contenus, ne me semblent peu ou pas assez enseignés. On s'y réfère très peu dans les formations. Je me souviens quand j'étais étudiant, lorsque la loi Sapin 1 est arrivée, tous les intervenants professionnels portaient le deuil. En gros, c'était castrateur. Ce n'était pas du tout perçu comme quelque chose de positif pour la profession. Quand on ne démontre pas que l'autorégulation peut être efficace, on se fait réguler par le législateur.

4. Evoquons votre expérience de formateur, comment former les étudiants à l'éthique en communication? Quelles sont les opportunités mais aussi les faiblesses d'une telle formation?

Je pense qu'il y a vraiment quelque chose à faire. J'ai l'impression que la génération actuelle est prête. En tout cas, j'ai pu observer les modèles qu'ils veulent suivre, les envies, leur fameuse «quête de sens », etc. Et j'ai l'impression que c'est un terreau fertile pour qu'ils entendent et valorisent les démarches éthiques. Les modèles valorisés à mon époque n'étaient pas exactement les mêmes. Nous avons une opportunité avec cette génération qui est en attente de ce type de contenu. Elle est en attente de sens, de savoir pourquoi elle fait ce job, de travailler sur et pour le « bien commun ». Comment le communicant peut définir son rôle en ayant un impact positif sur le bien commun? Cette génération semble être en attente de revaloriser de son métier. J'ai l'impression que cela ne relève pas simplement du fait d'être jeune, d'avoir un idéal, etc. Il me semble y avoir une vraie préoccupation éthique qui ne demande qu'à éclore.

Au cours de la formation, peut-on réussir à aborder les questions éthiques?

Oui, il y a tout d'abord un apprentissage un peu scolaire qui consiste à leur donner la boîte à outils car ça va les aider au quotidien dans leur premier stage ou leur première expérience professionnelle. Si ces jeunes arrivent à 23 ans dans une agence en disant qu'il faut être éthique... pas certain qu'ils soient écoutés. En revanche, s'ils 
sortent la charte ou le code UDA, COM-ENT, s'ils connaissent la loi Sapin 1 et 2, le RGPD, la norme ISO $26000 \ldots$ il y a de vraies chances qu'ils puissent faire changer les choses. Il faut les armer avec une bonne connaissance de l'ensemble de l'arsenal législatif et les référentiels issus des associations professionnelles, etc.

Après, ce que je fais dans mes cours, c'est de faire vivre l'éthique par les exemples et les exercices, c'est-à-dire que je leur donne à voir des pratiques non éthiques ou des case studies et je leur demande : face à cette situation, vous feriez quoi ? Et le débat s'instaure, les arguments sont échangés et chacun imagine les conséquences de ses actes. Concernant mon sujet «les études, les sondages » et leur communication notamment dans les médias, je les oblige à lire des méthodologies. Je les oblige à lire les private policy de Facebook pour voir ce qui se passe. Tout de suite, ça leur parle. Le principe c'est de les placer dans des situations très opérationnelles car l'éthique se vit au quotidien.

Notre éthique, à nous professionnels de la communication, est beaucoup plus terre à terre et sournoise que des cas de pots de vin par exemple. C'est vraiment leur faire toucher du doigt cette diversité des cas. Ce n'est pas nécessairement d'énormes sujets qui feront la Une du journal de $20 \mathrm{~h}$ mais derrière des actes qui semblent mineurs comme acheter des faux followers pour nourrir le fil twitter d'une entreprise, on va nourrir des mafias et faire travailler des enfants dans des pays lointains. Je les oblige à regarder en face ce qu'est le vrai, le faux, le gris. On est dans l'entre-deux. On travaille aussi le sujet sur les mots. On essaie de classer les mots entre sincérité, authenticité, transparence, vérité, etc. Y a-t-il une hiérarchie là-dedans ? Les entreprises peuvent-elles être transparentes ? Transparence veut-elle dire sincérité ?

On a également les cas Elise Lucet. Je les confronte à ça. Vous êtes communicants. Elise Lucet vous demande d'interviewer votre patron, que faites-vous? Comment faites-vous ? Et l'intérêt, c'est de débattre. Je leur prédis que dans les trois premières années de leur carrière, ils vont être confrontés à des cas où leur éthique sera questionnée. On parle aussi des objets connectés qui entrent dans nos univers, l'intelligence artificielle, les algorithmes, la neutralité du web, etc. Ils vont travailler sur tous ces sujets.

5. Dans votre dernier ouvrage «Big ou bug data? Manuel à l'usage des datadéontologues »1, vous abordez la question des data dans leur dimension éthique et défendez une déontologie du traitement des données, pouvez-vous nous présenter votre démarche?

'Adary, A. (2017). Big ou Bug Data? Manuel à l'usage des datadéontologues. Paris : Edition du Palio. 
Tout le débat sur les big data - à leur apparition, on parlait même d'évangélisation - s'est cristallisé sur les $4 \mathrm{~V}$ : la vitesse, la variété, le volume et la valeur. Au début, pendant des années, il n'y avait pas le 5ème $\mathrm{V}$ qui est celui de la vérité. L'idée de la démarche est de dire que les 4 premiers $V$, notamment celui de la valeur, ne valent rien s'il n'y a pas de véracité. Dans véracité, il y a la notion de juste propriété, la juste collecte (éthique de la collecte, de son analyse), etc. La France fait partie des meilleurs en matière de formation des data analyst, data scientist, etc. On devrait être capable de produire aussi des datadéontologues qui viendraient proposer, encapsuler l'ensemble des pratiques de data analyst pour leur donner un fondement d'éthique et de véracité. En fait, ce que je constate dans la pratique, c'est un peu comme des apprentis sorciers. On laisse les data analyst cruncher la data, collecter, etc. sans jamais s'interroger sur pourquoi on le fait. Est-ce qu'on a bien le droit de le faire ? Pour quelle finalité ? A la fin, ça produit des monstres. Et même si l'intention est bonne, l'usage des données peut donner lieu à des effets indésirables, des effets secondaires comme pour certains médicaments.

Si je me projette un peu dans le futur, si je regarde la masse de données qu'on va continuer à collecter notamment avec les objets connectés et si on tend vers des usages de plus en plus sérieux des data, de moins en moins récréatifs ou ludiques, il va falloir mettre une couche, une membrane de déontologie entre la production et l'analyse des données.

C'est-à-dire qu'un datadéontologue doit être « bilingue », capable de comprendre comment les données sont produites, la structure des algorithmes, leurs dimensions juridiques, avoir des connaissances sur le traitement, etc. et aussi être capable de parler avec les utilisateurs de la data, c'est-à-dire presque toutes les fonctions de l'entreprise (RH, DG, RSE, Commercial, etc.) : comprendre leur besoins, leurs usages. Dans cette relation, le datadéontologue doit être en capacité de faire converser l'ensemble des acteurs de la chaîne de valeur de la data.

Là, il y a un enjeu très fort et pas seulement juridique. Est-ce la loi, dans son acception terre à terre, pure et dure ou est-ce l'esprit des lois ? L'éthique est plutôt du côté de l'esprit des lois. C'est comme l'optimisation fiscale. Ce n'est pas illégal mais ce n'est pas moral. C'est un peu le même sujet avec mon datadéontologue, on pourrait imaginer des situations qui respectent scrupuleusement des textes de loi mais ce qu'on est en train de faire n'est pas éthique.

6. Justement, quels sont les enjeux et les risques liés au big data aujourd'hui ? Quels types de réponse envisager? Concernant la judiciarisation de la question avec notamment le RGPD 2018 ?

Ce que je vous disais au début, je trouve que les communicants ne se sont pas assez emparés de la communication responsable, de l'ISO 26000, etc. Là, je vois arriver le 
même problème avec le RGPD ${ }^{2}$. Il y a plein de directeurs de la communication qui vont me dire, ça c'est le sujet de la direction de la conformité, c'est le juridique, c'est le DSI qui s'en occupent et je leur dis non, non. Si on lit le texte du RGPD, on parle d'information, de transparence, d'obtenir le consentement des utilisateurs. Partout, partout, en filigrane, on parle de la communication et du communicant.

Le communicant est engagé dans ce sujet car, premièrement, sa fonction fabrique de plus en plus de data et de fichiers. Dans le RGPD, on parle de fichiers plus que de data. Le communicant est de plus en plus producteur de données. Deuxièmement, il va devoir gérer les risques réputationnels liés au RGPD. Je n'arrête pas de dire que le communicant doit avoir deux jambes : le verbe et le chiffre. Aujourd'hui, il faut absolument qu'il muscle sa compétence en chiffres. Il ne peut pas fuir dès qu'il y a un tableau excel.

Dans le RGPD, il y a le DPO, en français ils ont traduit par le Délégué à la Protection des Données, en anglais, data protection officer, il a un petit côté super héros, qui n'est pas très loin si je puis dire de mon datadéontologue mais qui est encore un peu trop du côté des juristes. C'est une vision assez stricte de la loi.

La force d'un règlement européen est qu'il est opposable. Il n'y a pas que les instances de régulation, type CNIL qui vont s'y intéresser, le citoyen, les ONG, les associations vont pouvoir se saisir de ce droit pour attaquer juridiquement. Les avocats disent que le risque d'amende, qui est très élevé, est très faible concernant les organismes de régulation. En revanche, les préjudices demandés par les citoyens et les consommateurs peuvent être très violents. Mais au-delà des amendes et des préjudices juridiques, le vrai risque est réputationnel. C'est pour cela que ça fait partie de l'éthique. Il n'est pas du côté du régulateur, qui va dire une ou deux fois, ce n'est pas bien... Ce sont les citoyens, les associations qui vont attaquer. Par exemple, si Uber annonce 1 mois après l'incident que 57 millions de comptes utilisateurs ont été piratés... je pense que dans le cadre du RGPD, ils peuvent avoir une class action de 57 millions de personnes.

Le dernier élément qui place le RGPD dans le giron de la communication, est un sujet de communication interne. Je pense qu'aujourd'hui, comme on a pu le faire pour les réseaux sociaux, il faut conduire de grandes démarches pour sensibiliser les collaborateurs, pour savoir ce qu'est un fichier, une donnée personnelle. Dans toutes les grandes entreprises, il y a une charte de bonnes pratiques concernant les réseaux sociaux, ce qu'on a le droit de dire ou pas, la confidentialité, etc. Il y a tout un pan de communication interne à mettre en place car aujourd'hui dans une entreprise de 5000 collaborateurs, cela ne m'étonnerait pas qu'il y a 5000 fichiers avec des données personnelles qui se promènent. Chacun refait son petit fichier. C'est comme ça qu'on voit sortir des fichiers avec une liste de collaborateurs et des commentaires

2 https://www.cnil.fr/fr/reglement-europeen-protection-donnees 
désobligeants associés. Le communicant ne pourra pas empêcher ces cas d'éthique personnelle mais il pourra sensibiliser à ce qu'est un fichier, que l'on ne peut pas créer de fichiers n'importe comment, que les risques de diffusion existent.

7. Dernière question, la revue Communication et professionnalisation s'intéresse particulièrement au processus de professionnalisation des communicateurs, comment voyez-vous se construire cette fonction que vous nommez «datadéontologue», quelles sont leurs responsabilités, les pratiques à développer?

J'ai été approché un moment donné par une institution de formation en me disant pourquoi on ne créerait pas une chaire de datadéontologie dans une école ? Pourquoi pas ! Et d'ailleurs, il faudrait qu'on enseigne quoi ? En effet, si on devait imaginer une formation et intégrer la datadéontologie dans les équipes communication, cela signifierait de professionnaliser les communicants sur le sujet des data, comprendre les data, leur fabrication, ne pas en avoir peur, les démythifier aussi, comprendre comment fonctionne un algorithme. Je ne dis pas avoir la compétence de le faire mais osons rentrer dans ces sujets. Pour moi, on ferait déjà un très gros saut de professionnalisation.

Ensuite, il y a tout ce que je décrivais pour les étudiants dans la professionnalisation de la fonction. Avoir pleinement conscience de tous les référentiels métiers sur l'éthique. Comprendre que ce sont nos amis et pas des empêcheurs de tourner en rond, pouvoir s'en servir, s'appuyer dessus. Donc, professionnaliser sur la base des référentiels. Après, je propose d'instaurer tous les semestres dans les équipes une « revue éthique » des pratiques comme ça se passe, je pense, dans le monde de la santé en hôpital. Être suffisamment en confiance pour dire voilà, j'ai fait ça, qu'en pensezvous? Est-ce bien ou pas bien? Parce que je pense vraiment que cela passe par la pratique, pour arriver à éradiquer les micro-pratiques non éthiques qui peuvent conduire à la fin à faire des choses monstrueuses.

Dire que la datatdéontologie est pleinement dans l'espace de la fonction communication, cela revient à résoudre deux difficultés : c'est dire que la data, c'est dans la communication et la déontologie, c'est dans la communication. Donc, cela génère un double bénéfice pour la fonction communication mais aussi deux axes de professionnalisation. 